\title{
Perceiving the Classical Cadence
}

\author{
David Sears, William E. Caplin, \& \\ STEPHEN McAdams \\ McGill University, Montreal, Canada
}

THIS STUDY EXPLORES THE UNDERLYING MECHANISMS responsible for the perception of cadential closure in Mozart's keyboard sonatas. Previous investigations into the experience of closure have typically relied upon the use of abstract harmonic formulæ as stimuli. However, these formulæ often misrepresent the ways in which composers articulate phrase endings in tonal music. This study, on the contrary, examines a wide variety of cadential types typically found in the classical style, including evaded cadences, which have yet to be examined in an experimental setting. The present findings reveal that cadential categories play a pivotal role in the perception of closure, and for musicians especially, ratings of the cadential categories provide empirical support for a model of cadential strength proposed in music theory. A number of rhetorical features also affect participants' ratings of closure, such as formal context, the presence of a melodic dissonance at the cadential arrival, and the use of a trill within the penultimate dominant. Finally, the results indicate that expertise modulates attention, with musicians privileging bassline motion and nonmusicians attending primarily to the soprano voice.

Received: June 22, 2012, accepted September 20, 2013.

Key words: cadence, closure, hierarchy, schema, Mozart

I N THE HISTORY OF MUSIC THEORY, THE CONCEPT of closure looms large. Given the seemingly exhaustive treatment this topic has received, particularly for music of the classical period (ca. 1775-1810), it is perhaps surprising to note its continued relevance in music scholarship. Consider the first eight measures from the third movement of Mozart's Piano Sonata in B-flat, K. 281, shown in Figure 1. With the exception of its somewhat unusual opening harmony (a tonicization of ii), this passage is in many respects a fairly conventional beginning in Mozart's keyboard style. Two phrases subdivide the opening eight measures: an antecedent phrase in mm. 1-4 followed by a consequent phrase in mm. 5-8. Although the second phrase largely repeats the first, a few compositional devices help to distinguish the consequent from the antecedent: the change from a piano to a forte dynamic, the subtle embellishments of the right-hand melody, and the expansion of register in the left hand. Yet, the most significant difference appears at the end of each phrase. The antecedent concludes with a dominant harmony in root position, a stable albeit active sonority whose metrical placement and expanded duration serve to reinforce the perception of ending. Theorists have termed this category of closure a half cadence. The ending of the consequent, and of the theme as a whole, features harmonic motion from a root-position dominant to a root-position tonic at the downbeat of $\mathrm{m} .8$, as well as the arrival of the melody on the first scale degree, characteristics that exemplify a category of closure theorists have termed a perfect authentic cadence.

Although we tend to theorize little as to how passages like this one begin, we have a great deal to say about how they end. The highly conventionalized nature of these endings has prompted theorists to describe and explain the compositional procedures involved in articulating phrase endings. The classical cadence perhaps best exemplifies this point, as it is a foundational concept in the Formenlehre tradition, and it continues to receive attention and undergo refinement in the scholarly community (for a review of theories of form in the history of music theory, see Burnham, 2002). Indeed, the revival of interest in theories of musical form over the last few decades has prompted a number of studies that reconsider previously accepted explanations of how composers articulate cadences in the classical period (Caplin, 2004), that classify instances in which cadential arrival fails to materialize (Caplin, 1998, pp. 101-11; Hatten 1992; Hepokoski \& Darcy, 2006, pp. 150-79; Schmalfeldt, 1992), and that attempt to situate the concept of cadence within a broader understanding of both tonal and formal closure (Anson-Cartwright, 2007; Blombach, 1987). Yet despite such intense theoretical scrutiny, it remains unclear how listeners represent these patterns in longterm memory, how cadences are perceived during music listening, and how the various features of cadences contribute to the perception of closure.

Although the compendium of cadences and other conventional closing patterns associated with the

Music Perception, volume 31, issue 5, pp. 397-417, issn 0730-7829, electronic issn 1533-8312. C 2014 by the Regents of the university of California All RIGHTS RESERVED. PLEASE DiRECT ALl REQUESTS FOR PERMisSion tO PHOTOCOPY OR REPRODUCE ARTICLE CONTENT THROUGH THE UNIVERSITY OF CALIFORNIA PRESS'S RIGHTS AND PERMISSIONS WEBSITE, HTTP://WWW.UCPRESSJOURNALS.COM/REPRINTINFO.ASP. DOI: 10.1525/MP.2014.31.5.397 

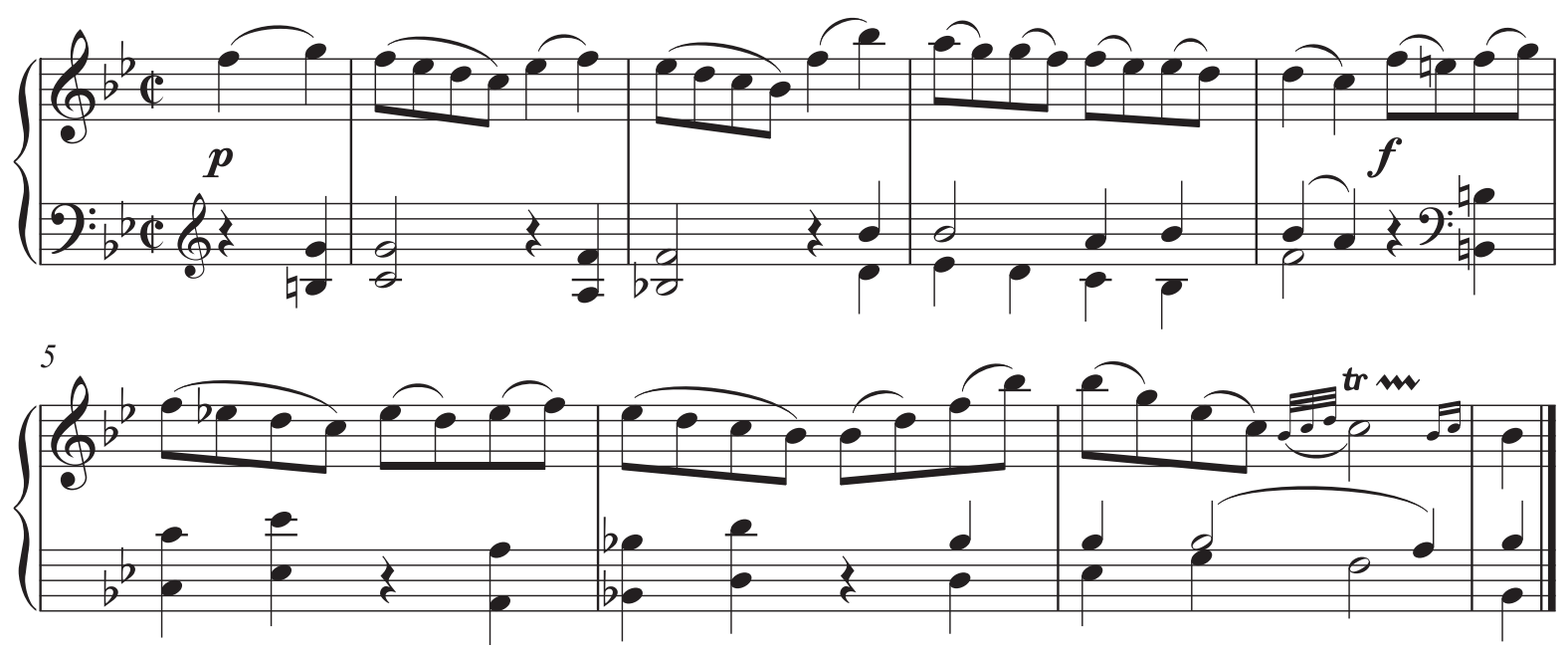

FIGURE 1. Wolfgang Amadeus Mozart, Piano Sonata No. 3, K. 281, iii, mm. 1-8.

classical period is enormous, contemporary scholars typically cite only a few, which may be classified according to two fundamental types: those cadences for which the goal of the progression is tonic (e.g., perfect authentic, imperfect authentic, deceptive, etc.), and those cadences for which the goal of the progression is dominant (e.g., half cadences). Moreover, whereas a number of parameters may contribute to the identification and categorization of cadences and other ending formulæ, theorists attend principally to melody and harmony, often referred to as among the syntactic parameters of tonal music (Agawu, 1987; Caplin, 2004; Meyer, 1989).

Figure 2 provides examples from Mozart's keyboard sonatas for the five most common cadential categories: perfect authentic (PAC), imperfect authentic (IAC), half (HC), deceptive (DC), and evaded (EV). Following the PAC category, the imperfect authentic cadence is typically identified as a melodic variant of the perfect authentic cadence, as it features an authentic cadential progression, but presents $\hat{3}$ in the melody at cadential arrival. Shown in Figure 2a-c, the PAC, IAC, and HC categories appear at the conclusion of phrases or entire themes and are typically followed by the initiation of a new formal process. Together, these three cadence categories constitute what Caplin (1998) has referred to as "the only genuine cadence categories in music in the classical style" (p. 43) because they are the only categories that can achieve thematic closure.

Whereas imperfect authentic and half cadences generally remain categorically distinct from the perfect authentic cadence, deceptive and evaded cadences do not. Instead, these two cadence categories arise as a result of the failed attempt to attain authentic cadential closure. Furthermore, these cadential deviations typically only affect the final tonic of the cadential progression at the moment of the cadential arrival. In the deceptive cadence, the final root-position tonic is replaced by some other harmony, usually VI (see Figure 2d). Yet because a deceptive cadence features a melodic line that resolves to a stable scale degree at the cadential arrival (typically to $\hat{1}$ or $\hat{3}$ ), it still provides a provisional sense of ending. Finally, shown in Figure 2e, the evaded cadence is characterized by the complete failure of the final harmonic and melodic events of the cadence to materialize. Instead, the event that appears in place of the expected cadential goal represents a "new beginning." Evaded cadences typically feature a sudden interruption in the projected resolution of the melodic line to 1 . Instead, the melody leaps up, often to $\hat{5}$, thereby replacing the expected ending with material that initiates the subsequent phrase. Thus, the evaded cadence provides no sense of ending whatsoever.

In the classical style, the aesthetic effect of these cadential deviations is undeniable, as instances of cadential deception and evasion serve to intensify the expectation for authentic cadential closure at the ends of movements or larger sections. In the exposition section of a sonata-form movement, for example, Schmalfeldt (1992) has argued that the emergence of the subordinate (or secondary) theme in compositional practice coincided with the increasing tendency to highlight the cadential progression as the ultimate signal for thematic closure (p. 1). Thus, the subordinate theme is often characterized by the appearance of numerous deviations and extensions of the authentic cadential progression that serve to delay the moment of cadential arrival 

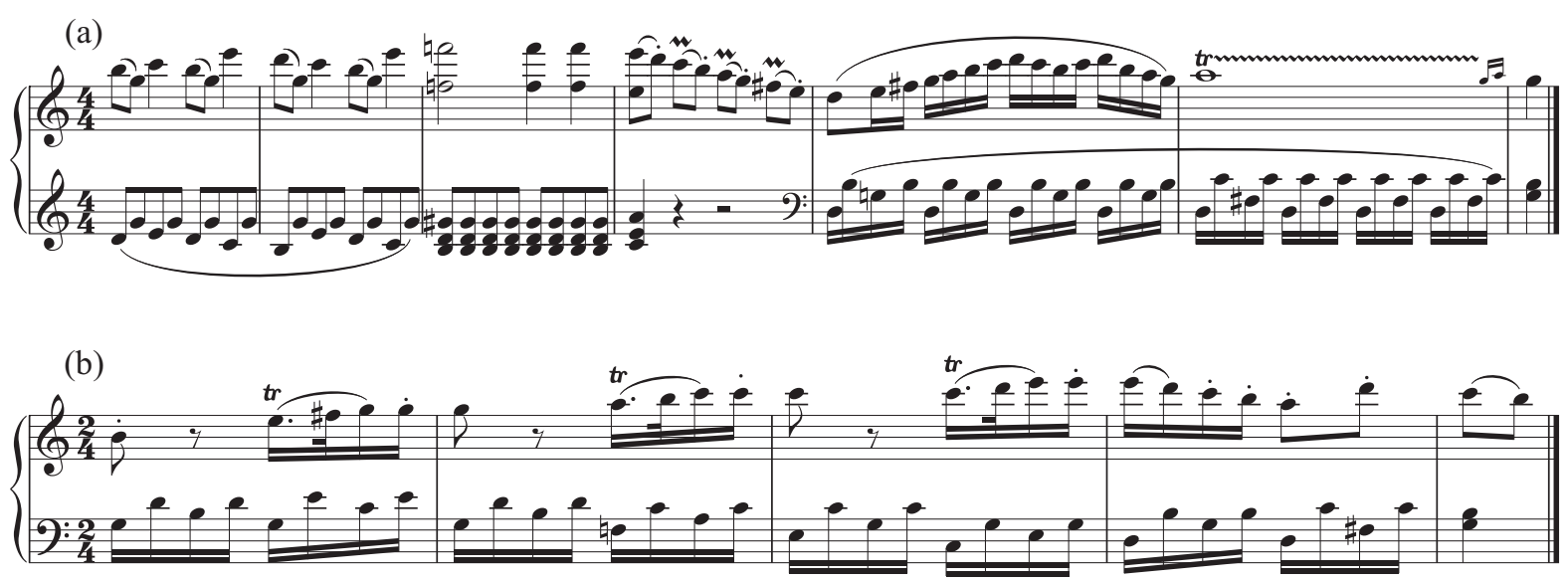

(c)

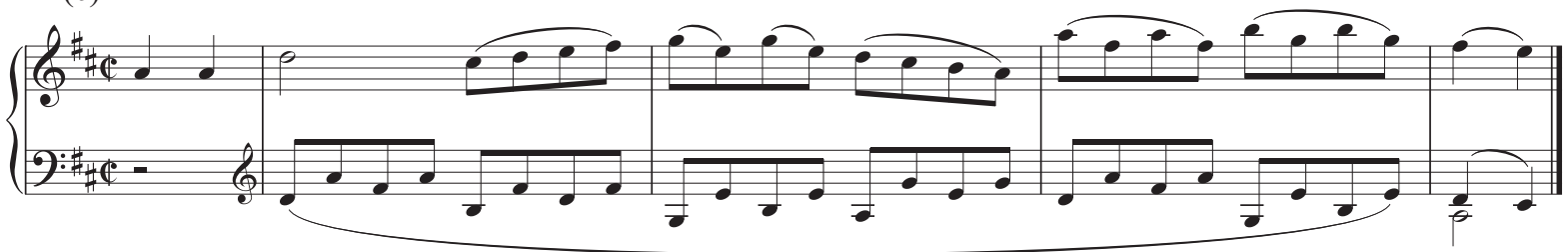

(d)

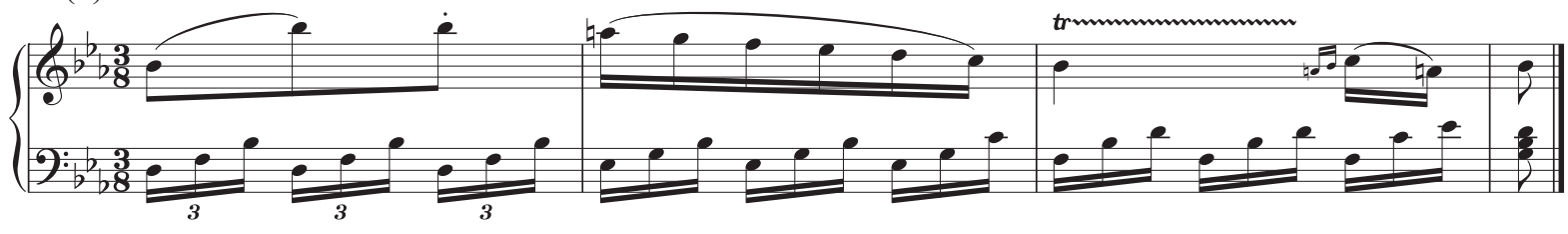

(e)

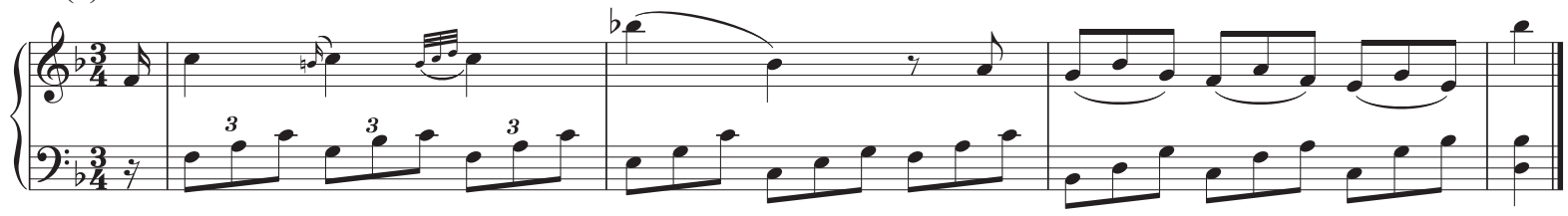

FIGURE 2. Five excerpts representing the five cadential categories. (a) PAC Category, Subordinate Theme Subtype: K. 309, i, mm. 48-54. (b) IAC Category, Melodic Dissonance Subtype: K. 330, iii, mm. 39-43. (c) HC Category, Main Theme Subtype: K. 284, iii, m1-4. (d) DC Category, Failed PAC Subtype: K. 281, ii, mm. 32-35. (e) EV Category, Non-Tonic Subtype: K. 279, ii, mm. 1-4.

(Caplin, 1987; 2000; Hatten, 1992; Schmalfeldt, 1992), and number of authors have noted the expressive and formal implications of dramatizing cadential arrival in this manner (Caplin, 1998; Hepokoski \& Darcy, 2006; Schmalfeldt, 1992).

Many theorists have therefore accounted for the perception of closure by appealing to theories of expectation (Huron, 2006; Meyer, 1956; Narmour, 1989; 1992), as cadential progressions elicit very definite expectations concerning the melodic scale degree, the harmony, and the metric position of the goal event. The formation and violation of these expectations has been empirically tested in continuation and expectancy ratings tasks for melodies (Cuddy \& Lunney, 1995; Schellenberg, 1996; 1997; Schmuckler, 1989; 1997), in melodic priming paradigms (Aarden, 2003; Margulis \& Levine, 2006), in harmonic priming (Bharucha \& Stoeckig, 1986; Bigand \& Pineau, 1997; Bigand, Madurell, Tillmann \& Pineau, 1999; Justus \& Bharucha, 2001; Tillmann \& Bigand, 2001), and in harmonic priming during melodies (Loui 
\& Wessel, 2007). Moreover, several studies have employed authentic cadential (V-I) progressions explicitly to examine harmonic expectations using behavioral, (Loui \& Wessel, 2007), neural (Janata, 1995; Koelsch et al., 2003; Koelsch, Fritz, Schulze, Alsop \& Schlaug, 2005; Koelsch, Gunter, Friederici \& Schröger, 2000; Koelsch, Schmidt \& Kansok, 2002; Leino, Brattico, Tervaniemi \& Vuust, 2007; Loui, Grent-'t-Jong, Torpey \& Woldorff, 2005; Maess, Koelsch, Gunter \& Friederici, 2001; Tillmann, Janata \& Bharucha, 2003), and psychophysiological measures (Steinbeis, Koelsch \& Sloboda, 2006).

The evidence is thus overwhelming that expectation plays a significant role in the cognitive processing of harmony and melody, parameters that remain essential to the perception of cadential closure. Furthermore, cadences and other ending patterns have frequently been employed as stimuli to investigate more general cognitive processes-for example, how listeners store harmonic and tonal structure in long-term memory (Krumhansl \& Kessler, 1982) or the perception of dynamic variations in tension (Bigand \& Parncutt, 1999). It should be no surprise, then, that in many of these studies, the perception of closure plays a prominent role. Indeed, Aarden (2003) has noted an unintended effect of closure in Krumhansl's highly influential work probing the tonal hierarchy (Krumhansl, 1990; Krumhansl \& Kessler, 1982). In a study comparing the goodness-of-fit ratings she obtained for the 12 members of the chromatic scale with the frequency-of-occurrence of these scale degrees in various corpora from Western music, Krumhansl has claimed that listeners form a cognitive representation of the tonal hierarchy by internalizing the distributional properties of tonal music. Noting a disparity in Krumhansl's results, ${ }^{1}$ however, Aarden conducted a reaction-time experiment designed to test the assumption that scale degrees receiving a higher "fit" rating in Krumhansl's tonal hierarchy would lead to faster reaction times. Yet, the results of the experiment only confirmed this hypothesis when Aarden asked participants to respond exclusively to the final event of each melody, leading him to conclude that the probe-tone method employed by Krumhansl and Kessler actually encouraged listeners to treat the probe tone as a phrase-final event. Thus, Aarden claimed,

\footnotetext{
${ }^{1}$ Although the correlations between the scale-degree distributions of various corpora with Krumhansl's goodness-of-fit ratings are quite high $(r>.80)$, several discrepancies remain unexplained, the most noteworthy example being that the dominant scale degree normally appears more frequently than the tonic degree in the various corpora, yet in Krumhansl and Kessler's key profiles, the tonic receives the highest "fit" rating.
}

Krumhansl and Kessler's major and minor key profiles reflect a cognitive representation of the tonal hierarchy that pertains specifically to endings. Furthermore, Krumhansl, Bharucha and Kessler (1982) noted a harmonic closure effect for two-chord progressions selected from the chords of the diatonic scale, as the relatedness judgments obtained for these progressions revealed a hierarchy of stability (I, V, IV, VI, II, III, VII), with more stable chords serving as better continuations in the two-chord context. Noting that stable harmonies like I and V typically received higher continuation judgments when they followed, rather than preceded, the remaining diatonic harmonies, Bharucha and Krumhansl (1983) further proposed that stable tones and chords appear at the ends of phrases because they serve as cognitive reference points, an expression coined by Rosch (1975) for elements that are characterized by their asymmetric temporal relations with less stable elements. Indeed, Tan, Aiello, and Bever (1981) have suggested that listeners use stable harmonies like I and V to segment melodic phrases, even in the absence of an explicit harmonic context, and Cuddy, Cohen, and Mewhort (1981) have noted that melodies were better remembered if they contained a strong tonal ending.

From an examination of the literature, it appears that cadences play a vital role in the perception of tonal music (Bigand \& Parncutt, 1999). It is by no means contentious, moreover, that internal representations of the tonal and harmonic hierarchies affect the perception of closure, but it remains far less clear whether listeners possess schematic representations for various melodic and harmonic closing formulæ. Boltz (1989) has reported effects of serial order on the perception of melodic closure, but the issue as to whether listeners store melodic closing patterns in long-term memory remains open. Yet for bass-line motion, the serial position of the events preceding a phrase ending is fundamental to current definitions of cadential closure. The cadential status of the final tonic in an authentic cadence, for example, is crucially determined by the harmony of the preceding event. In a study examining the perception of harmonic closure in a two-chord context, Rosner and Narmour (1992) reported that schematic representations of root progressions common to known cadences appeared to play the most prominent role in explaining the participants' ratings, leading the authors to claim that the various harmonic formulæ located at phrase endings result in the formation of schematic representations of harmonic closure. Eberlein and Fricke (1992) have extended this claim to the melodic, rhythmic, and metric parameters employed in the articulation of cadences, theorizing that experienced listeners 
of tonal music form schematic representations for frequently occurring cadential formulæ.

What remains absolutely essential to such a claim, however, is that the strength of the schematic representation depends on a listener's exposure to a given musical style. A growing body of evidence reveals that listeners develop implicit knowledge of tonal and harmonic structure simply as a result of passive exposure to Western music. The psychological reality of Krumhansl's tonal and harmonic hierarchies therefore reflects a general purpose cognitive learning mechanism, in which knowledge about the external environment is acquired without conscious awareness (Reber, 1989). Researchers have since extended this claim to explain how listeners process harmony and melody, proposing a connectionist framework to account for Western harmonic syntax (Bharucha, 1987; Bharucha \& Stoeckig 1986; Tillmann, Bharucha \& Bigand, 2000) and employing artificial grammars to examine how listeners respond to novel harmonic and melodic contexts (Jonaitis \& Saffran, 2009; Rohrmeier, Rebuchat, \& Cross, 2011; Tillmann \& Poulin-Charronnat, 2010). Moreover, children appear to develop a sensitivity to harmonic structure at around 5 to 7 years of age, even in the absence of explicit formal training (Corrigall \& Trainor, 2010; Trainor \& Trehub, 1994). Researchers have also reported harmonic priming effects in children between 5-11 years of age using both behavioral and neural measures (Koelsch et al., 2003; Schellenberg, Bigand, Poulin-Charronnat, \& Stevens, 2005), and Costa-Giomi (2003) has even extended this claim explicitly to the perception of cadences, suggesting that by 6 years of age, children notice the lack of a conclusive cadence if it is missing from a progression, and by 8 they can discriminate between conclusive and inconclusive cadences.

Unfortunately, the effect either of explicit music training or of passive exposure on the perception of closure still remains unclear: several authors have reported significant effects of musical expertise for a variety of cadential stimuli (Eberlein \& Fricke, 1992; Vallières, 2011; Vallières, Tan, Caplin, \& McAdams, 2009; Vos \& Pasveer, 2002; Weiser, 1992), whereas others have reported no effects whatsoever (Boltz, 1989; Tillman, Bigand, \& Madurell, 1998). Such contradictory reports as to the role of explicit formal training or implicit learning on the perception of closure may reflect differences either in the choice of experimental task or in the use of stimuli, as researchers often prefer to employ abstract cadential paradigms rather than examples of cadences from genuine musical literature. And there are certainly very good reasons for doing so: by eliminating variations in dynamics, tempo, and rhythm, as well as disregarding a number of features that appear frequently in compositional practice (e.g., the cadential six-four, the suspension dissonance at cadential arrival, or a melodic trill at the penultimate dominant, henceforth termed the "cadential trill"), such abstract paradigms provide greater experimental control and are much easier to alter to satisfy specific experimental needs. But these paradigms also misrepresent the ways in which composers often articulate phrase endings in tonal music (and consequently the ways in which listeners might actually perceive these endings), as they ignore many of the features of cadences that might contribute to the perception of closure. Indeed, whereas theorists attend principally to the syntactic parameters of tonal music, in compositional practice each cadence may be realized in nearly countless ways, entailing parameters of rhythm, meter, texture, and instrumentation. Thus, cadences also differ as a result of their unique nonsyntactic or rhetorical content (Agawu, 1987; Caplin, 2004), an issue that has not been considered in an experimental setting. In Mozart's keyboard style, for example, cadences may also be characterized according to the formal context from which they were drawn (e.g., main theme, transition, subordinate theme, etc.) or by the presence of a melodic dissonance at the cadential arrival.

The recent revival of interest in the Formenlehre tradition in music scholarship has also largely gone unnoticed in the music psychology community, as those studies explicitly examining the perception of closure rarely employ the wide variety of cadential types found in the "common practice" period. Techniques for cadential deviation, in particular, serve an important formal and expressive function in the classical style, but they have yet to be considered in an experimental setting. By using examples drawn from real music, the experimental study of cadential failure could serve to explore rich areas of inquiry in music psychology, such as the perception of closure, the processing of harmonic syntax, and the generation and violation of expectations. Finally, examining a variety of cadential categories will provide the opportunity to consider the cadential hierarchy. Rosner and Narmour (1992) have suggested that style-structural closing schemata may be stored hierarchically in long-term memory, and several theorists have proposed hierarchical models of cadential strength (Caplin, 1998; Latham, 2009; Schmalfeldt, 1992), yet the psychological reality of the cadential hierarchy has not been examined empirically.

The following experiment attempted to address these issues directly. Although an exploration of the underlying 
cognitive mechanisms responsible for the perception of closure in tonal music is the ultimate aim of this research, our initial approach is more limited in scope, concentrating as it does on a closing pattern that appears frequently in tonal music: the classical cadence. From Mozart's keyboard sonatas, we selected fifty excerpts that contained an equal number of perfect authentic (PAC), imperfect authentic (IAC), half (HC), deceptive (DC), and evaded cadences (EV). These categories were chosen both on the basis of their frequency in Mozart's style as well as on their assumed relevance to research in music theory and music perception. We hypothesized that participant ratings would significantly differ among the categories and that the melodic and harmonic content specific to each category would play the most substantial role in participants' ratings of closure.

To consider the effects of additional parameters within the cadence categories, each category was subdivided into two subtypes in order to examine more closely parameters not embraced by category membership, such as formal context (for the PAC and HC categories), the presence of a melodic dissonance at the cadential arrival (for IAC and HC), and the melodic scale degree and harmony at the cadential arrival (for DC and EV, respectively). The intent here was to consider compositional parameters that could play an important role in the perception of closure, as well as to attempt to select subtypes that reflect the most prevalent features of Mozart's compositional style.

Each perfect authentic cadence was classified according to its location within an exposition section of sonata form, as closing either a main theme or a subordinate theme. The excerpts selected from the subordinate theme further feature an expanded cadential progression (ECP) (see Figure 2a), which, in addition to its longer duration, is characterized by a dramatic increase in surface activity and the appearance of a cadential trill (for a discussion of the ECP, see Caplin, 1987). The imperfect authentic cadence category was subdivided according to the presence or absence of a melodic dissonance at the cadential arrival. Figure $2 \mathrm{~b}$ presents the former case, in which the melodic goal of the cadential arrival (B5) is embellished by an accented passing tone (C6). Although a number of other features might serve to differentiate imperfect authentic cadences, such as the metric placement of the cadential arrival (i.e., "masculine" vs. "feminine" endings) or the contour of the melody preceding cadential arrival (ascending vs. descending), the appearance of a surface dissonance in the melody is an especially prominent attribute of Mozart's imperfect authentic cadences. As with the PAC category, half cadences were also classified according to their formal location, as either from a main theme (shown in Figure 2c) or a transition. The material within the transition in sonata form typically modulates to the key of the subordinate theme and often features increased surface activity. This increased energy and tonal instability might serve to differentiate half cadences ending a transition from those found in a main theme. In addition, excerpts from the HC category were also separately classified according to the presence or absence of a melodic dissonance at cadential arrival. (Excerpts containing a dissonance are marked with an asterisk in Table 1.) The deceptive cadence category was subdivided according to the melodic scale degree (indicated by the caret) present at the cadential arrival: $\hat{1}$, which we refer to as a failed perfect authentic cadence (as in Figure $2 \mathrm{~d}$ ), and $\hat{3}$, referred to as a failed imperfect authentic cadence. $^{2}$ Finally, the evaded cadence category was subdivided according to the harmony that appears in place of the cadential arrival-tonic harmony, which may be in root position or inverted, or non-tonic harmony (as in Figure 2e).

Although the cadential subtypes permit an extended examination of the role played by parameters within each cadence category, these subtypes still fail to consider the relative contribution of a variety of additional parameters that appear frequently in cadential contexts (e.g., the presence of a cadential trill, the use of a dissonant six-four chord suspended above the cadential dominant, the temporal duration of the cadential progression, etc.). Thus, in addition to the subtypes, we also characterized each excerpt according to a number of rhetorical parameters in order to examine their relative contribution in a regression model.

\section{Method}

\section{PARTICIPANTS}

Participants were 40 members (19 male) of the Montreal community recruited through the Schulich School of Music and the McGill University classified ads. Ages ranged from 18 to $48(M=24, S D=6)$. Twenty participants with music training equivalent or superior to second-year-university level formed the musician group, and twenty participants with less than one year

\footnotetext{
${ }^{2}$ Unfortunately, the subtypes for the HC and DC categories do not contain an equal number of excerpts. For the HC category, the main theme subtype contains four, whereas the transition subtype contains six. However, the surface dissonance subtypes for the HC category contain an equal number of excerpts. Additionally, because deceptive cadences are fairly uncommon in Mozart's keyboard sonatas, the failed perfect authentic cadence subtype includes seven, whereas the failed imperfect authentic cadence subtype includes three.
} 
TABLE 1. Cadence Categories, Syntactic Characteristics, Subtypes, and Reference Information (Köchel index, movement, measures) for each Excerpt.

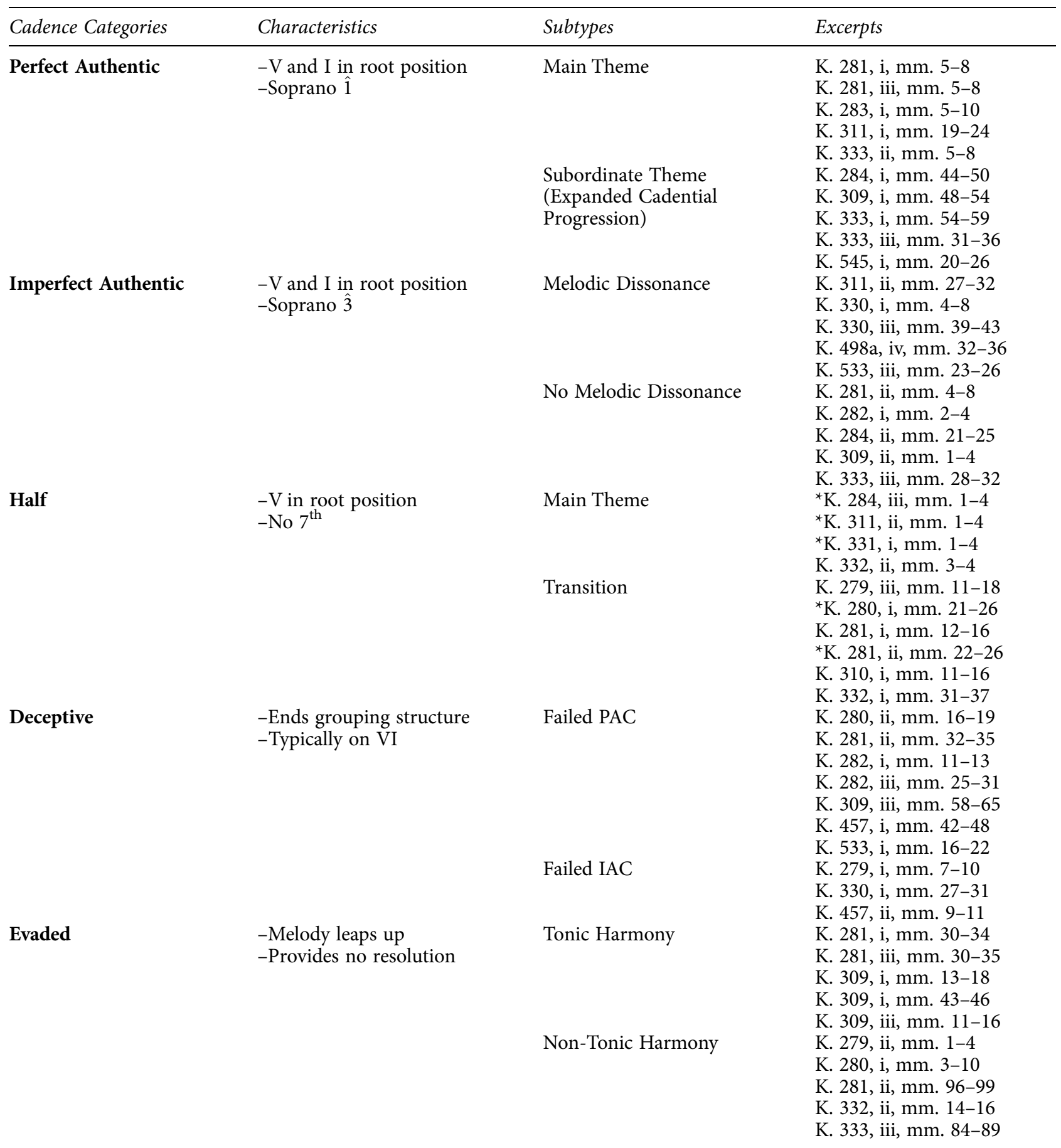

Note. Excerpts from the HC category marked with an asterisk contain a surface dissonance at cadential arrival, whereas the remaining excerpts from that category do not.

of music training comprised the nonmusician group. To limit any effects caused by familiarity with the stimuli, no participant with more than one year of formal study on the piano was permitted to take part.
A questionnaire was administered to assess musical preferences and training. On average, musicians had 11.4 years of study on a musical instrument (other than piano), 3.5 years of ear training, 3.0 years of instruction 
in harmony, and 2.9 years of instruction in music analysis. At the time of their participation, they additionally reported spending an average of 15.2 hours each week engaged in instrumental practice. Participants also listened to an average of 11.3 hours of music each week. All of the participants reported normal hearing, which was confirmed with a standard audiogram administered before the experiment (ISO 398-8, 2004; Martin \& Champlin, 2000), and none of the participants indicated they had absolute pitch.

\section{MATERIALS}

The stimuli consisted of 50 short excerpts (average $8.6 \mathrm{~s}$ ) selected from Mozart's keyboard sonatas; the excerpts contained an equal number of perfect authentic cadences, imperfect authentic cadences, half cadences, deceptive cadences, and evaded cadences. It is also important to note that every excerpt in this study contained a cadential progression-a functional harmonic progression that precedes (and includes) the goal harmony (Caplin, 2004). Consequently, the cadential categories in this stimulus set differ from one another primarily as a result of the pitch content located at the moment of cadential arrival (or, in the case of the evaded cadences, the moment of anticipated arrival). To limit the number of variables under consideration, performance features (such as dynamics and rubato) were neutralized and the tempo of each excerpt was determined by convention. Each stimulus was first created with the notation software Finale and then realized as a .wav sound file using a piano physical model created by PianoTeq. Finally, a 4-s fade-in was inserted at the beginning of each excerpt to encourage participants to attend specifically to the excerpt's end.

Unfortunately, the extraction of each excerpt from its surrounding material raised several issues associated with the moment of the cadential arrival. In an effort to eliminate some of these unwanted effects while still preserving the stylistic integrity of each excerpt, it was necessary to impose a few constraints on the cadential arrival. First, any chord tones appearing after the cadential arrival (e.g., an Alberti bass pattern) were verticalized to the moment of the cadential arrival, and all subsequent material was removed. This step eliminated differences in surface activity among excerpts, in particular where the absence of the third of the triad would have resulted in an unstylistic open octave. Second, the duration of the cadential arrival was recomposed to one full tactus to ensure that differences in duration did not affect the perception of closure. Third, since this study did not consider the effect of cadential absence (such as when a rest replaces the expected tonic at the cadential arrival), in two instances the events following the rest were shifted back to the cadential arrival.

DESIGN AND PROCEDURE

The participants were seated in a double-walled IAC Model 1203 sound-isolation chamber. The stimuli were reproduced on a Macintosh G5 PowerPC, output as S/PDIF using an M-Audio Audiophile 192 sound card, converted to analog using a Grace Design m904 monitor system, and presented over a pair of Dynaudio BM6A monitors. The stimuli were presented at $55 \mathrm{~dB}$ SPL, which was kept constant for all participants throughout the experimental session. The experimental program, subject interface, and data collection were programmed using the Max/MSP environment from Cycling 74' controlled by the PsiExp software environment (Smith, 1995).

Participants were presented with a randomized set of all 50 excerpts in two blocks. After listening to each excerpt up to three times, participants were instructed to rate the degree of completion of each excerpt on a 7-point continuous analogical-categorical scale (Weber, 1991), which consists of an analog scale subdivided into seven discrete categories labeled from 1 to 7 . Completion was defined as: "the expectation that the music will not continue. A value of 1 indicates that the excerpt would certainly continue, whereas a value of 7 indicates that the excerpt could end at that moment without the need for anything further." Participants were encouraged to use the full range of the scale over the course of the experiment. In addition, at no point was the term cadence ever mentioned during the session under the assumption that its usage might unintentionally bias musicians toward consciously categorizing the excerpts' endings.

In addition to a completion judgment, participants rated on 7-point scales the confidence of their completion rating and their familiarity with the excerpt. To distinguish between those excerpts potentially rated in the center of the completion scale, the participants also responded to the following two statements on a 4-point Likert scale labeled from strongly agree to strongly disagree: "this excerpt could complete an entire work or movement," and "this excerpt could complete a phrase or short passage of music." The aim of the additional 4-point Likert response scales was to ask participants to differentiate between endings located at the conclusion of a longer work from those endings they may deem sufficient to complete a phrase or short passage within that work, a distinction assumed too subtle to be captured by the completion scale, particularly for those excerpts that participants placed in the center of 


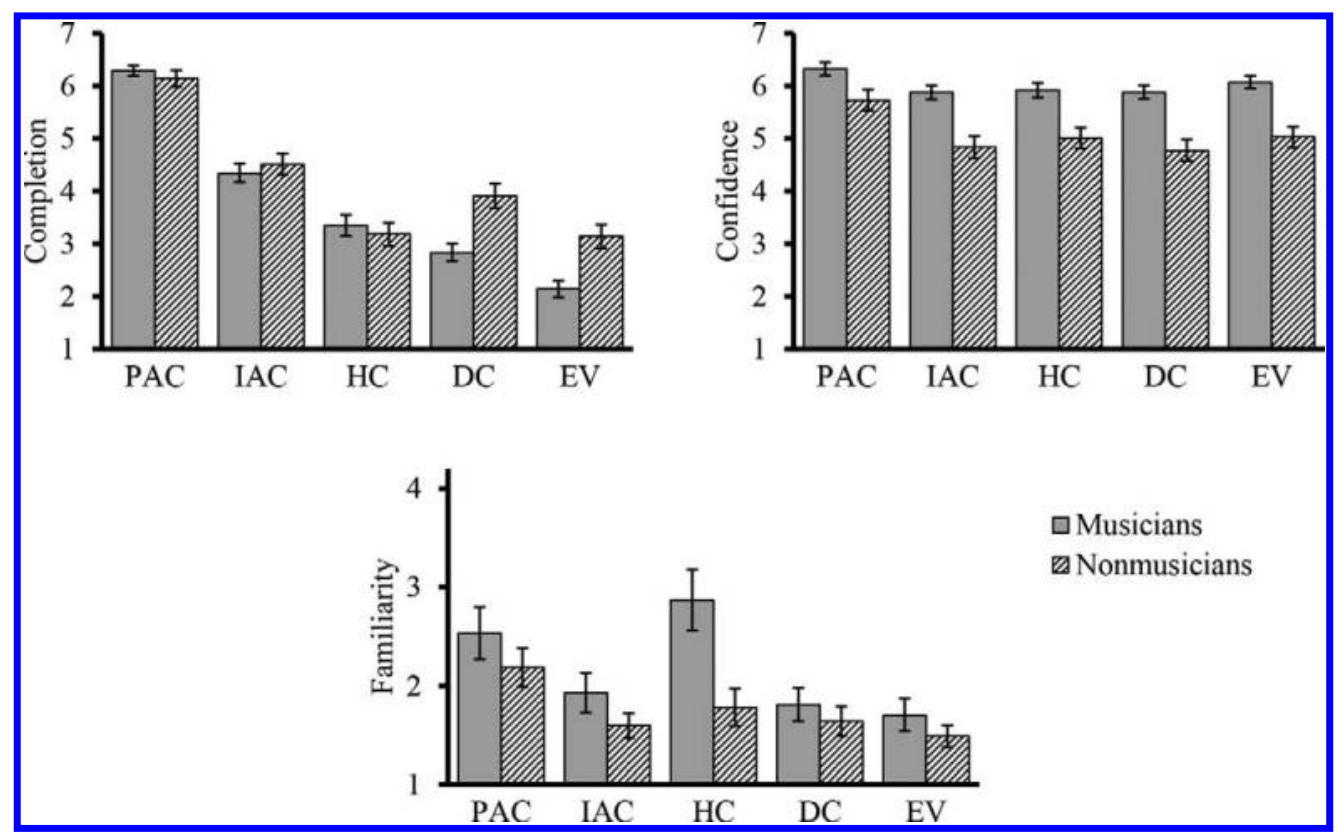

FIGURE 3. Bar plots of mean completion, confidence, and familiarity ratings of musicians and nonmusicians for each cadential category. Whiskers represent the $95 \%$ confidence interval. See text for the abbreviations of the cadence categories.

the scale (i.e., excerpts rated as neither entirely complete nor entirely incomplete). The participants were also reminded that the two scales should not necessarily co-vary. By strongly agreeing that an excerpt could complete an entire work or movement, a participant might also assume it could complete a phrase or short passage of music. The reverse is not necessarily true, however, because an excerpt might provide an entirely implausible ending for an entire work, yet sound satisfactory at the end of a short passage within the work.

To familiarize the participants both with the range of stimuli as well as with the experimental task, the experimental session began with an exposure phase consisting of ten additional excerpts (two excerpts exemplifying each cadence category), and a practice phase in which the participants rated each of the ten excerpts. After completing the experiment, participants filled out a short questionnaire addressing their music background. ${ }^{3}$

\footnotetext{
${ }^{3}$ Because the behavioral scales for the completion, confidence, and familiarity ratings are bounded on both sides (by 1 and 7), in a few cases the aggregated data for the perfect authentic and evaded cadential categories-the categories expected to elicit very high and very low completion ratings, respectively-violated assumptions of normality and homogeneity of variance due to their skewed distributions. To eliminate these issues before calculating the model, the completion, confidence, and familiarity ratings were normalized to the range [0-1] and an arcsin transformation was applied. In the figures that follow, however, the raw data were retained for the purposes of visualization. To counteract
}

\section{Results}

\section{CADENCE CATEGORIES}

Figure 3 displays bar plots of the completion, confidence, and familiarity ratings for each of the five cadential categories. A mixed $5 \times 2$ ANOVA of the completion ratings revealed main effects of cadential category, $F(3.14$, $119.27)=264.56, \varepsilon=0.79, p<.001, \eta^{2}=.85$, and music training, $F(1,38)=8.39, p<.01, \eta^{2}=.18$, as well as a significant interaction, $F(3.14,119.27)=8.52, p<.001$, $\eta^{2}=.03$. For the musician group, post hoc analyses revealed significant differences between each pair of cadential categories descending from PAC to EV $(p<.01)$, with the exception of a marginal difference appearing for the HC-DC pair $(p=.06)$. The membership of each excerpt to a cadential category therefore appeared to significantly affect the completion ratings for the musician group. Musicians and nonmusicians did not differ in their ratings for any of the categories of genuine cadences (PAC, IAC, HC), but the results did reveal an effect of music training for the failed cadences (DC, EV), as nonmusicians provided higher completion ratings for both deceptive and evaded cadences than did musicians $(p<.001)$. Indeed, nonmusicians did not rate half

violations of sphericity, degrees of freedom are reported using the Greenhouse-Geisser correction where appropriate. Finally, all post hoc statistics were conducted using $t$-tests with Bonferroni adjustment. 


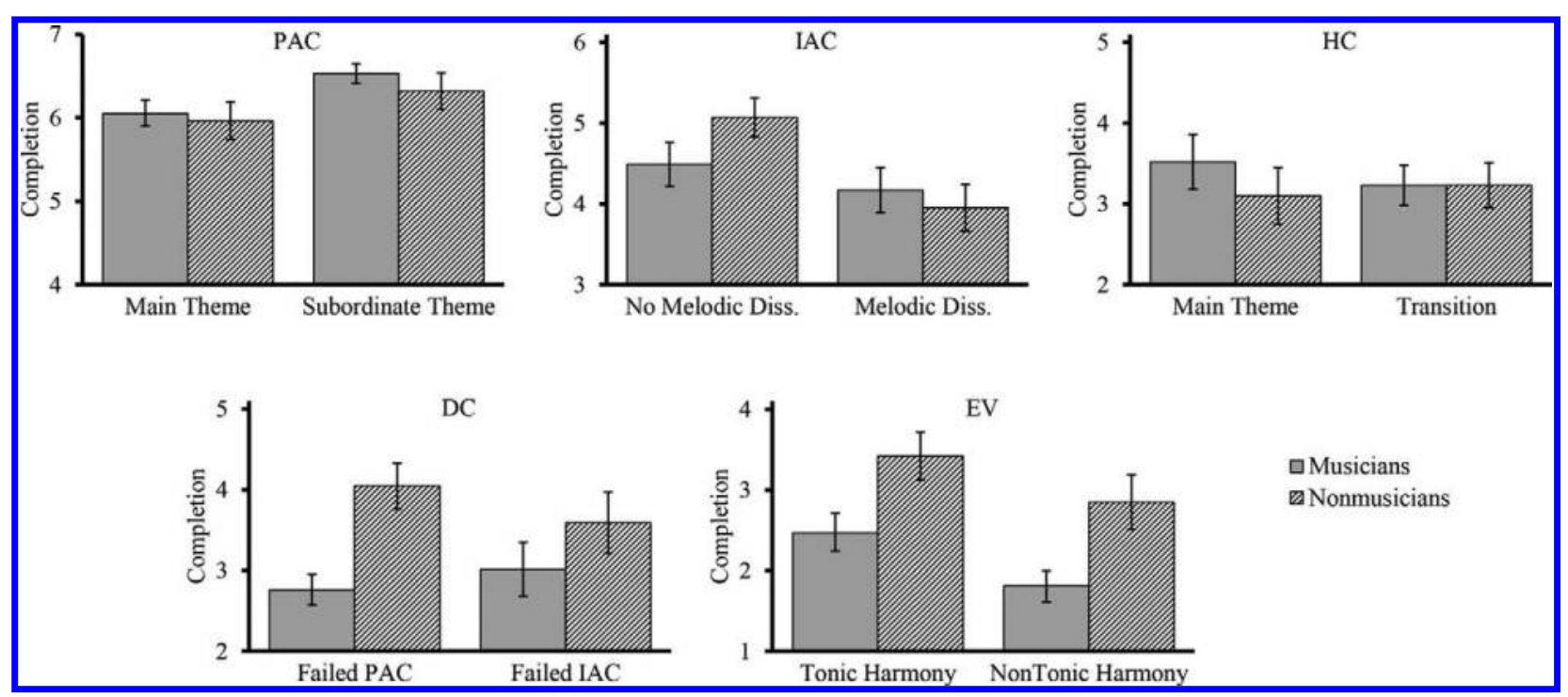

FIGURE 4. Bar plots of mean completion ratings of musicians and nonmusicians for each of the ten subtypes. $Y$-axes present a range of 3 units within the scale [1-7]. Whiskers represent the $95 \%$ confidence interval. See text for the abbreviations of the cadence categories.

cadences as any more complete than evaded cadences $(p>.05)$.

As expected, confidence ratings averaged across participants were weakly correlated with the completion ratings, Spearman's rank correlation coefficient $r_{\mathrm{s}}(50)=.36, p=.01$. A mixed $5 \times 2$ ANOVA revealed a significant effect of cadence category, $F(2.52,95.75)=$ 25.44, $\varepsilon=0.63, p<.001, \eta^{2}=.39$, as both groups provided higher confidence ratings for excerpts from the PAC category than for any of the other cadence categories $(p<.01)$. Confidence ratings were also higher for musicians than for nonmusicians, $F(1,38)=18.93$, $p<.001, \eta^{2}=.33$. Although both groups provided low familiarity ratings on average, completion ratings were nonetheless weakly correlated with familiarity, $r_{\mathrm{s}}(50)=$ $.50, p<.001$. Familiarity judgments also revealed main effects of cadential category, $F(2.95,112.01)=34.23$, $\varepsilon=0.74, p<.001, \eta^{2}=.41$, and training, $F(1,38)=$ $4.47, p=.04, \eta_{\mathrm{p}}{ }^{2}=.11$, as well as an unexpected interaction resulting from differences in the way musicians and nonmusicians specifically rated half cadences, $F(2.95,112.01)=12.26, p<.001, \eta^{2}=.14$. As shown in Figure 3, whereas both groups provided higher familiarity ratings for the perfect authentic cadence category than for the other categories $(p<.05)$, musicians also rated excerpts from the half cadence category as more familiar than those from the remaining categories $(p<.05)$. This effect was not observed in nonmusicians, however, as they provided very low familiarity ratings for excerpts ending in half cadences, nor did these ratings differ from those of the other categories. The intention behind providing a scale for familiarity was to determine if explicit prior exposure to a particular excerpt would affect completion ratings, assuming that knowledge of the material following the end of the excerpt might alter the interpretation of that excerpt's ending, thus confounding the experimental outcome. However, the observed difference in familiarity ratings of excerpts ending with half cadences between musicians and nonmusicians instead suggests a difference in their exposure to, and subsequent knowledge of, half cadences in general, a particularly compelling finding that appears to contradict the completion data, in which no training effect was observed for half cadences.

\section{CADENCE SUBTYPES}

Figure 4 presents bar plots of the completion ratings for the cadential subtypes of each cadential category. Beginning with the perfect authentic cadence category, both groups rated PACs selected from the subordinate theme as more complete than PACs from the main theme, $F(1,38)=23.43, p<.001, \eta^{2}=.38$, and there was no effect of training, $F(1,38)<1$. For the imperfect authentic cadence category, the presence of a melodic dissonance at the cadential arrival only affected completion ratings for nonmusicians $(p<.01)$. In addition, nonmusicians provided higher completion ratings for imperfect authentic cadences without a dissonance at the cadential arrival than did musicians $(p<.05)$. For the half cadence category, musicians tended to rate half 


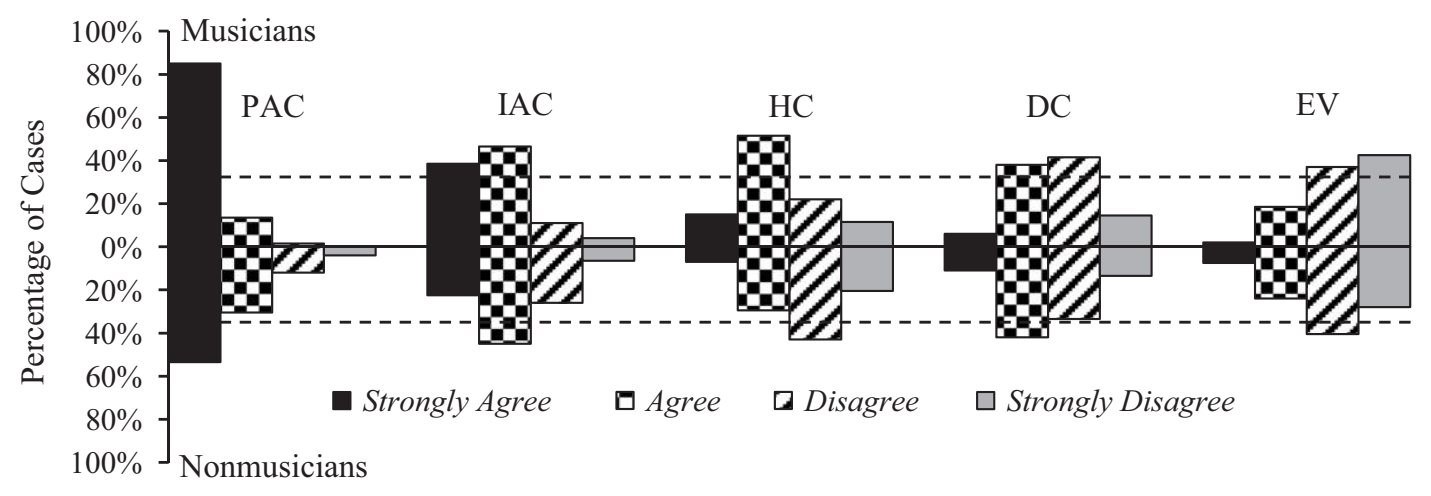

FIGURE 5. Bar plot of the distribution of the percentage of responses for each cadential category for the statement, "this excerpt could complete a phrase or short passage of music," with musicians' ratings above and nonmusicians' ratings below the $\mathrm{x}$-axis. The dotted line indicates the minimum threshold necessary to reach significant agreement.

cadences selected from transitions as less complete than those selected from the main theme, but this interaction between formal context and music training did not reach significance, $F(1,38)=3.10, p=.08$, nor did the completion ratings differ with respect to training, $F(1,38)<1$. However, excerpts from the HC category were also classified according to the presence or absence of a melodic dissonance at cadential arrival, and for both groups excerpts that contained a melodic dissonance received significantly lower completion ratings than excerpts that did not, $F(1,38)=4.5, p<.05$, $\eta^{2}=.15$, and there was no effect of training, $F(1,38)<1$.

The completion ratings for the deceptive cadence category revealed a significant effect of expertise, $F(1,38)=24.0, p<.001, \eta^{2}=.39$, and the scale degree at the cadential arrival significantly affected the completion ratings of nonmusicians $(p<.05)$, as they provided higher ratings for deceptive cadences featuring melodic $\hat{1}$ than for those featuring melodic $\hat{3}$. However, the scale degree at the cadential arrival did not affect the ratings of the musician group. Finally, the evaded cadence category featured a significant effect of expertise, $F(1,38)=28.16, p<.001, \eta^{2}=.43$, with musicians providing much lower completion ratings for evaded cadences than nonmusicians. Additionally, the harmony at the cadential arrival significantly affected the completion ratings of both participant groups, as evaded cadences with tonic harmony at the cadential arrival received higher completion ratings than cadences with non-tonic harmony $(p<.05)$.

\section{MOVEMENT COMPLETION AND PHRASE COMPLETION RATINGS}

The movement ratings provided very few notable results, as both participant groups featured significant interparticipant agreement for the majority of the excerpts, and in nearly all of those cases they strongly disagreed that the excerpt could complete a work or entire movement. However, the phrase ratings revealed a significant effect of music training for the half cadence category. Figure 5 provides a bar plot of the distribution of the percentage of responses for each cadential category for the statement, "this excerpt could complete a phrase or short passage of music," with musician ratings above and nonmusician ratings below the $\mathrm{x}$-axis. This representation therefore visually estimates the similarity between the two groups by evaluating the symmetry about the $\mathrm{x}$-axis. A chi-square test was performed to determine the minimum number of trials necessary to reach significant agreement for each category. Thus, out of 200 trials within each category (20 participants $\times$ 10 excerpts in each cadence category), a minimum of 68 identical responses (or 34\%) was necessary to achieve significance, $\chi^{2}(1)=3.89, p<.05$. The horizontal dotted lines above and below the $\mathrm{x}$-axis indicate this minimum agreement threshold. The very first category in the musician group, for example, indicates that in $85 \%$ of all trials musicians strongly agreed that excerpts from the PAC category could complete a phrase or short passage of music. For the IAC and HC categories musicians generally agreed with this statement, although they generally disagreed that excerpts from the EV category could complete a short passage. Concerning the DC category, both groups wavered between agree (40\%) and disagree $(37.5 \%)$, and although musicians minimally preferred to disagree with the statement whereas nonmusicians preferred to agree, this difference was not significant, Mann-Whitney $U=18,017, p=.067, r=$ -.09. Indeed, whereas both groups differed with respect to the absolute percentage of agreement of their responses, the shape of the distribution for each cadential category remained fairly similar between the two groups. In the case of the $\mathrm{HC}$ category, however, the 
TABLE 2. Descriptive Statistics for the 12 Rhetorical Features.

\begin{tabular}{|c|c|c|c|}
\hline Rhetorical Features & $M(S D)$ & Range & Mode (Frequency) \\
\hline \multicolumn{4}{|l|}{ Entire Stimulus } \\
\hline (1) Tempo (bpm) & $104(31)$ & $40-152$ & \\
\hline (2) Event Density & $6.3(2.6)$ & $2.7-13.3$ & \\
\hline (3) Median Pitch Height (MIDI note number) & $68(5)$ & $58-81$ & \\
\hline (4) Stimulus Duration (s) & $9.6(2.3)$ & $5.6-16.8$ & \\
\hline \multicolumn{4}{|l|}{ Cadential Progression } \\
\hline (5) Complete & & & Present (30) \\
\hline (6) Cadential Trill & & & Absent (33) \\
\hline (7) Cadential ${ }_{4}^{6}$ & & & Present (38) \\
\hline (8) Cadential Progression Duration (s) & $3.9(2.3)$ & $0.9-12.2$ & \\
\hline \multicolumn{4}{|l|}{ Cadential Arrival } \\
\hline (9) Melodic Dissonance & & & Absent (39) \\
\hline (10) Metric Downbeat & & & Present (42) \\
\hline (11) $\Delta$ Event Density & $6.7(5.9)$ & $2-23$ & \\
\hline (12) Tactus Duration (s) & $0.8(0.5)$ & $0.2-2.3$ & \\
\hline
\end{tabular}

${ }^{\mathrm{a}}$ Event Density refers to the number of notes per second.

${ }^{\mathrm{b}}$ Complete refers to an authentic cadential progression that includes an initial tonic, a pre-dominant, a dominant, and a final tonic or to a half cadential progression that includes an initial tonic, a pre-dominant, and a dominant.

${ }^{\mathrm{c}}$ Melodic Dissonance may refer to an appoggiatura, an accented passing tone or a dissonant suspension.

${ }^{\mathrm{d}} \Delta$ Event Density was calculated as the difference between the sum of the events in a 1-s window preceding cadential arrival to the sum of the events in a $1 \mathrm{~s}$ window beginning at cadential arrival.

difference in the responses of musicians and nonmusicians was significant, $U=13,923, p<.001, r=-.28$; in over $66 \%$ of their responses musicians agreed or strongly agreed that a half cadence could complete a phrase or short passage, whereas nonmusicians disagreed or strongly disagreed in $63 \%$ of their responses.

\section{RHETORICAL FEATURES}

The purpose of the preceding analysis was to determine the role various cadential categories might play in the perception of closure. To that end, five distinct cadential categories were selected on the basis of their melodicharmonic content. But in selecting examples from the extant musical literature, the stimuli fail to control for a number of rhetorical parameters that may affect participants' ratings of completion (e.g., tempo, event density, a cadential trill, etc.). Indeed, the syntactic characteristics that distinguish the cadence categories employed in this study are frequently accompanied by a number of other features that may also facilitate the identification of cadences. A trill, for example, may serve as a contextual cue that alerts the listener to an impending cadential ending. The following analysis therefore considers the degree to which these rhetorical parameters might predict the participant completion ratings. Unfortunately, the small number of stimuli employed for this study (50) prohibits establishing a reliable multiple linear regression (MLR) model embracing the vast number of musical parameters implicated in the articulation of cadences. Nonetheless, this correlational approach may lead to the identification of a small number of parameters $(k<6)$ to be examined in future studies (Miles \& Shevlin, 2001).

By considering rhetorical parameters, our assumption was that the cadence categories alone would not account for all of the variance in the completion ratings. Thus, before encoding the rhetorical parameters, it was necessary to determine the effect of cadence category membership in a regression model. Each excerpt's cadential category was encoded along an ordinal scale from PAC to $\mathrm{EV}$, following the ranking displayed in the musician completion ratings (see the completion ratings in Figure 3). This ranking accounts for $84 \%$ of the variance in the mean completion ratings from the musician group (standardized coefficient $\beta=.92$ ). However, the ranking only explains roughly $53 \%$ of the variance in the nonmusician completion ratings $(\beta=.73)$. This difference in the estimates of the MLR models may result from differences in selective attention during the perception of closure, or it may suggest that rhetorical parameters have a more significant impact on nonmusicians.

To consider the role these rhetorical parameters might play, 12 features were selected that characterize (1) the entire stimulus, (2) the cadential progression, and (3) the moment of cadential arrival (see Table 2):

1. Four features characterize the entire stimulus: the tempo in beats per minute (Tempo), the total number of notes per second (Event Density), the 
median pitch height in MIDI note values (Median Pitch Height), and the duration of the stimulus in seconds (Stimulus Duration).

2. Three dichotomous features and one continuous feature characterize the cadential progression: the presence of every harmonic function within the boundaries of the cadential progression ( $\mathrm{Com}$ plete), the presence of a cadential six-four (Cadential $\left.\frac{6}{4}\right)$, a trill within the cadential dominant (Cadential Trill), and the duration of the cadential progression in seconds (Cadential Progression Duration).

3. Two dichotomous features and two continuous features characterize the cadential arrival: the presence of a surface dissonance in the melody (Melodic Dissonance), the metric location of the final harmony, which can occur either on or off the downbeat (Metric Downbeat), the change in event density at the cadential arrival ( $\Delta$ Event Density), and the duration of the events at the cadential arrival (Tactus Duration).

To limit the number of predictors input into the final MLR models, correlations were calculated for each of the rhetorical features with the completion ratings from both groups. Shown in Table 3, intercorrelations between the rhetorical features displayed very few noteworthy results, with only three correlation coefficients registering above .50. Two features in particular, Complete and Cadential Trill, were not correlated with any of the other rhetorical features. Moreover, the majority of the moderate-to-strong correlations shown in Table 3 arose as a result of features that characterize temporal aspects of the stimulus. For example, stimulus tempo was correlated with the duration of the tactus at cadential arrival, $r(50)=-.46$, as well as of the stimulus as a whole, $r(50)=-.36$. However, the correlations between Melodic Dissonance and two other features-Metric Downbeat and Tactus Duration-were noteworthy, as excerpts that contained a melodic dissonance at cadential arrival also tended to emphasize the dissonance by lengthening the duration of cadential arrival and placing it on a metric downbeat, a result that suggests a compositional strategy to accentuate the effect of the dissonance.

The musician completion ratings were significantly correlated with four rhetorical features-Median Pitch Height, Complete, Cadential Trill, and $\Delta$ Event Density -and the nonmusician ratings were correlated with two features-Median Pitch Height and Cadential Trill. However, these correlations could simply result from their collinearity with a third variable, the rank order of cadential categories. It was therefore necessary to control for cadence category membership first by calculating the correlation between the completion ratings and the rank order of cadential categories, and then correlating each of the rhetorical features with the residuals. Controlling for the effect of cadential category resulted in significant correlations for four features with the musician ratings-Melodic Dissonance, semi-partial correlation coefficient $\operatorname{sr}(50)=.43, \Delta$ Event Density, $s r(50)=.40$, Cadential Trill, $s r(50)=.35$, and Cadential Progression Duration, $s r(50)=.26$-and four features with the nonmusician ratings-Melodic Dissonance, $s r(50)=.55$, Stimulus Duration, $s r(50)=.30$, Cadential Progression Duration, $\operatorname{sr}(50)=.28$, and Cadential Trill, $s r(50)=.28$.

Given the small number of remaining features, a stepwise MLR model with forward selection was fitted for both the musician and nonmusician completion ratings using the cadential ranking $\mathrm{PAC}>\mathrm{IAC}>\mathrm{HC}>\mathrm{DC}>\mathrm{EV}$ and the rhetorical features provided above. To examine effects of multicollinearity within the two models, correlations were calculated for all of the predictors. Of the five predictors input in the two models, only two-the cadential ranking and Cadential Trill-were weakly correlated, $r(50)=.30, p<.05$. Moreover, the mean Variance Inflation Factors calculated for the predictors in the musician (mean VIF $=1.10)$ and nonmusician (mean VIF $=1.02)$ models demonstrated very weak multicollinearity.

Shown in Table 4, the musician model selected the cadential rankings, Melodic Dissonance, Cadential Trill, and $\Delta$ Event Density. These four predictors accounted for $91 \%$ of the variance in the completion ratings. However, the combined size of the effect for the selected rhetorical features was small $\left(\Delta R^{2}=.07\right)$, thereby suggesting that the cadential categories play the most substantial role in the musicians' ratings. The nonmusician model selected the cadential rankings, Melodic Dissonance, and Stimulus Duration, and these three predictors accounted for $72 \%$ of the variance in the completion ratings. Moreover, the addition of Melodic Dissonance and Stimulus Duration significantly improved the fit of the model $\left(\Delta R^{2}=.19\right)$, indicating that the two rhetorical features played a more substantial role in the nonmusician model than those selected in the musician model.

By retaining the cadential categories, the above models unfortunately fail to consider how features of the soprano and bass voice may independently contribute to the perception of closure, particularly at the moment of cadential arrival. The claim that strategies of attention may differ as a result of music training therefore necessitates 


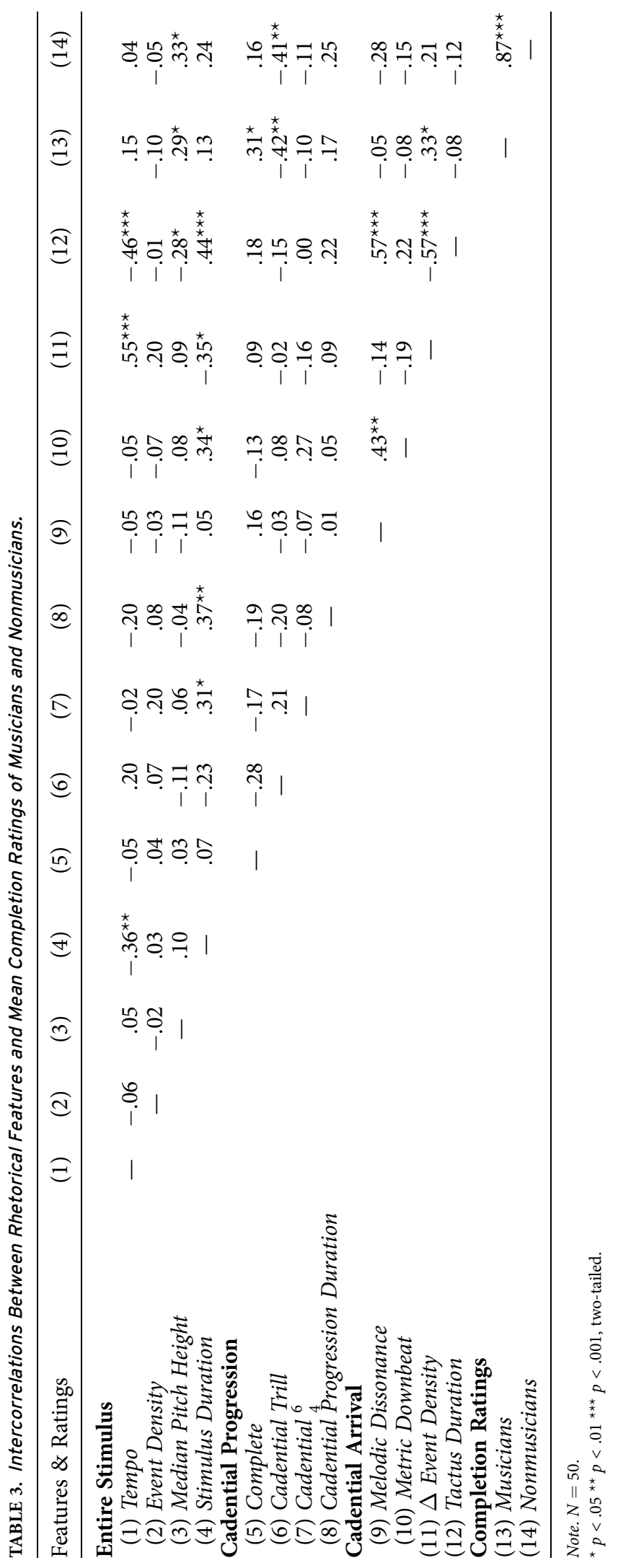


TABLE 4. Summary of Stepwise Regression Analysis Predicting the Completion Ratings of Musicians and Nonmusicians with the Cadential Rankings and the Correlated Rhetorical Features from Table 2.

\begin{tabular}{|c|c|c|c|c|}
\hline & & $B$ & $S E B$ & $\beta$ \\
\hline \multicolumn{5}{|l|}{ Musicians } \\
\hline & Step 1 & & & \\
\hline & Constant & 0.85 & 0.21 & \\
\hline & $\mathrm{PAC}>\mathrm{IAC}>\mathrm{HC}>\mathrm{DC}>\mathrm{EV}$ & 0.98 & 0.06 & $.92^{\star *}$ \\
\hline & \multicolumn{4}{|l|}{ Step 2} \\
\hline & Constant & 0.27 & 0.26 & \\
\hline & $\mathrm{PAC}>\mathrm{IAC}>\mathrm{HC}>\mathrm{DC}>\mathrm{EV}$ & 1.01 & 0.06 & $.94^{* *}$ \\
\hline & Melodic Dissonance & 0.64 & 0.19 & $.18^{\star}$ \\
\hline & \multicolumn{4}{|l|}{ Step 3} \\
\hline & Constant & 0.75 & 0.28 & \\
\hline & $\mathrm{PAC}>\mathrm{IAC}>\mathrm{HC}>\mathrm{DC}>\mathrm{EV}$ & 0.96 & 0.06 & $.90^{* *}$ \\
\hline & Melodic Dissonance & 0.64 & 0.18 & $.17^{\star}$ \\
\hline & Cadential Trill & 0.49 & 0.16 & $.15^{\star}$ \\
\hline & \multicolumn{4}{|l|}{ Step 4} \\
\hline & Constant & 0.68 & 0.26 & \\
\hline & $\mathrm{PAC}>\mathrm{IAC}>\mathrm{HC}>\mathrm{DC}>\mathrm{EV}$ & 0.92 & 0.05 & $.86^{* *}$ \\
\hline & Melodic Dissonance & 0.54 & 0.17 & $.15^{\star}$ \\
\hline & Cadential Trill & 0.51 & 0.15 & $.16^{\star}$ \\
\hline & $\Delta$ Event Density & 0.04 & 0.01 & $.15^{\star}$ \\
\hline \multicolumn{5}{|l|}{ Nonmusicians } \\
\hline & \multicolumn{4}{|l|}{ Step 1} \\
\hline & Constant & 2.20 & 0.30 & \\
\hline & $\mathrm{PAC}>\mathrm{IAC}>\mathrm{HC}>\mathrm{DC}>\mathrm{EV}$ & 0.67 & 0.09 & $.73^{* *}$ \\
\hline & \multicolumn{4}{|l|}{ Step 2} \\
\hline & Constant & 1.12 & 0.35 & \\
\hline & $\mathrm{PAC}>\mathrm{IAC}>\mathrm{HC}>\mathrm{DC}>\mathrm{EV}$ & 0.71 & 0.08 & $.78^{\star *}$ \\
\hline & Melodic Dissonance & 1.19 & 0.26 & $.38^{\star *}$ \\
\hline & \multicolumn{4}{|l|}{ Step 3} \\
\hline & Constant & -0.10 & 0.53 & \\
\hline & $\mathrm{PAC}>\mathrm{IAC}>\mathrm{HC}>\mathrm{DC}>\mathrm{EV}$ & 0.70 & 0.07 & $.77^{\star *}$ \\
\hline & Melodic Dissonance & 1.22 & 0.25 & $.39^{*}$ \\
\hline & Stimulus Duration & 0.12 & 0.04 & $.23^{*}$ \\
\hline
\end{tabular}

Note. Musicians $R^{2}=.84$ for Step $1 ; \Delta R^{2}=.03$ for Step 2 ( $\left.p s<.01\right) ; \Delta R^{2}=.02$ for Step $3(p s<.01) ; \Delta R^{2}=.02$ for Step $4(p s<.01)$. Nonmusicians $R^{2}=.53$ for Step $1 ; \Delta R^{2}=.14$ for Step 2 ( $p s<.01) ; \Delta R^{2}=.05$ for Step 3 ( $p s<.01$ ).

${ }^{*} p<.01{ }^{* *} p<.001$.

a statistical approach in which the melodic and harmonic information of each excerpt is encoded separately, thereby permitting us to abandon the cadential categories proper.

In order to characterize the events in the soprano and bass voices, the mean goodness-of-fit ratings obtained from Krumhansl and Kessler's (1982) major and minor key profiles were assigned to the scale degrees appearing at the cadential arrival: these profiles are thus assumed to signify a cognitive representation of the tonal hierarchy pertaining specifically to endings (Aarden, 2003). Presumably the metric position and rhythmic duration of the final events also affect participant ratings of completion, but in only 8 of the 50 stimuli did the cadential arrival appear in a metric position other than the downbeat (see Table 2). Moreover, the rhetorical feature measuring the duration of the events at cadential arrival, called Tactus Duration, was not significantly correlated with the participant completion ratings. In noncadential contexts, these variables might play an important role in the perception of closure, but in these stimuli, the metric position and rhythmic duration of the events concluding each stimulus could not be examined in greater detail.

Before entering the tonal stability values of the soprano and bass voices as predictors in a regression model, the two variables were correlated to determine if they violated the assumption of multicollinearity. In this instance, the variables were not correlated, $r(50)=-.07$, $p=.33$. Table 5 displays the model estimates. For the musicians' ratings, the tonal stability of the bass voice played the most prominent role, with a final $\beta$ of .73, followed by the soprano voice in the second step. Thus, as predicted, musicians appeared to place greater 
TABLE 5. Summary of Stepwise Regression Analysis Predicting the Completion Ratings of Musicians and Nonmusicians with the Tonal Stability Estimates of the Soprano and Bass Voices at Cadential Arrival.

\begin{tabular}{llrrr}
\hline & & $B$ & SE B & $\beta$ \\
\hline Musicians & & & & \\
& Step 1 & & & \\
$\quad$ Constant & -1.10 & 0.73 & \\
& $\quad$ Bass Tonal Stability & 0.93 & 0.14 & $.70^{*}$ \\
& Step 2 & & & \\
$\quad$ Constant & -4.16 & 0.71 & \\
& Bass Tonal Stability & 0.97 & 0.10 & $.73^{*}$ \\
& Soprano Tonal & 0.58 & 0.89 & $.49^{*}$ \\
& Stability & & & \\
Nonmusicians & & & \\
& Step 1 & & & \\
$\quad$ Constant & 1.12 & 0.57 & \\
& Soprano Tonal & 0.63 & 0.11 & $.62^{*}$ \\
& Stability & & & \\
& Step 2 & & & \\
$\quad$ Constant & -2.63 & 0.60 & \\
$\quad$ Soprano Tonal & 0.67 & 0.08 & $.66^{*}$ \\
$\quad$ Stability & & & \\
$\quad$ Bass Tonal Stability & 0.68 & 0.08 & $.60^{*}$ \\
\hline
\end{tabular}

Note. Musicians $R^{2}=.49$ for Step $1 ; \Delta R^{2}=.24$ for Step $2(p s<.001)$. Nonmusicians $R^{2}=.39$ for Step $1 ; \Delta R^{2}=.36$ for Step $2(p s<.001)$.

${ }^{*} p<.001$.

emphasis on the bass voice at the cadential arrival. For the nonmusician group, the tonal stability of the soprano voice was selected in the first step, with a $\beta$ of .66. However, the difference in $\beta$ weights in the final model was marginal $(\Delta \beta=.06)$, suggesting that nonmusicians were sensitive to variations in both voices at the cadential arrival.

\section{Discussion}

The goal of this study was to explore the underlying mechanisms responsible for the perception of cadential closure in Mozart's keyboard sonatas. The present findings indicate that, regardless of training, listeners appear to differentiate among the categories of genuine cadences (PAC, IAC, HC). The harmonic and melodic content at the cadential arrival (i.e., the syntactic parameters of tonal music) therefore plays a pivotal role in the perception of closure. Moreover, both groups provided higher completion, confidence, and familiarity ratings for the PAC category than for the other categories, providing converging evidence in support of the claim that listeners familiar with Western music possess a schematic representation for authentic cadential closure (Eberlein, 1997; Eberlein \& Fricke, 1992; Gjerdingen, 2007; Rosner \& Narmour, 1992; Sloboda, 1985).
From the inclusion of subtypes within each category, a number of conclusions may be drawn. First, the formal context of the PAC category significantly affected the perception of completion, regardless of music training, with excerpts drawn from subordinate themes receiving higher completion ratings. Perfect authentic cadences ending subordinate themes exhibit a number of unique characteristics that might explain this result: the expanded duration of the cadential progression, the increased surface activity (usually in the form of an accompanimental Alberti bass), and the appearance of a melodic trill just prior to the cadential arrival. Recent evidence also suggests that the sudden decrease in surface activity at the cadential arrival may affect the perception of closure (Vallières, 2011, p. 106).

Second, several results from the completion ratings for subtypes of the IAC and DC categories suggest that nonmusicians attend predominantly to the melody when assessing the completion of a given excerpt. First, they provided much higher completion ratings than did musicians for deceptive cadences, a cadential category that features a significant deviation from the expected harmony at the cadential arrival. Second, they were more sensitive to the presence of a surface dissonance in the melody at the cadential arrival, as evidenced by their lower ratings for that subtype of the imperfect authentic cadence category. Finally, differences in the melodic scale degree in the deceptive cadence category significantly affected the ratings of nonmusicians, with melodies featuring $\hat{1}$ receiving higher ratings than those featuring $\hat{3}$, a result that was not replicated in the ratings of the musician group.

Contrary to the nonmusician group, musicians appeared to be much more sensitive to variations in harmony at the cadential arrival, as they provided much lower completion ratings for deceptive cadences than did nonmusicians. Moreover, the harmony at the cadential arrival in the evaded cadence category also significantly affected musicians' ratings. Thus, the observed results might suggest a difference in attending strategies, with melody playing a more prominent role for nonmusicians, harmony a more prominent role for musicians.

Indeed, the regression estimates for the rhetorical features and tonal stability values strengthen this claim, as both the Melodic Dissonance feature and the tonal stability values for the soprano voice played a more substantial role in the nonmusician models. This finding supports Weiser's (1992) claim that music training may modulate attention, whereby nonmusicians demonstrate an attentional bias toward the soprano voice and musicians appear to flexibly track between the soprano and bass voices (pp. 40-46). There has been some empirical 
support for the claim that nonmusicians privilege parameters related to melodic motion, such as pitch proximity and contour, whereas musicians attend principally to harmonic factors, such as the interval size between two events (Vos \& Pasveer, 2002). Furthermore, Loui and Wessel (2007) have shown that these differences of attention might not be conscious. In a selective attention task, the authors asked participants to respond to the contour of melodies supported by harmonic progressions that were either highly expected, slightly unexpected or extremely unexpected. Their results suggested that, even when presented with a task that explicitly directed participants to attend to the contour of the melody, violations in harmonic expectancy still influenced the behavioral responses of musicians. Because this effect was not observed in nonmusicians, the authors claimed that repeated exposure to Western music results in the formation of automatic expectations to harmonic progressions that musicians simply cannot ignore, even when asked to attend to other features of the stimulus.

Given the emphasis placed on the bass voice in identifying and categorizing cadences in music theory, the musician estimates for the tonal stability values in the bass and soprano voices are therefore not unexpected. It remains unclear, however, whether attention to bassline motion in cadential contexts reflects a flexible voice-tracking strategy promoted during explicit formal training (i.e., in a pedagogical context) or an attentional bias formed simply through implicit exposure to Western music, a distinction that requires further attention in the experimental literature (Bigand, 2003). What these results do suggest is that, when faced with an explicit completion task, musicians appear to privilege the bass voice, whereas nonmusicians appear to be more sensitive to subtle differences in the soprano voice.

However, the hypothesis that differences in the completion ratings for the failed cadence categories may result from differences in attending strategy does not explain the significantly lower familiarity ratings from the nonmusician group for half cadences, nor does it explain why nonmusicians generally disagreed with the statement that half cadences could complete a phrase or short passage of music. Indeed, the completion ratings also suggest a different ordinal ranking of the cadential strength of each category for the two groups. The musician group provided the highest completion ratings for the PAC category, followed by the IAC, HC, DC, and EV categories, and this ranking provides empirical support for a model of hierarchical cadential closure first proposed by Caplin (1998). For the nonmusicians, however, the HC category did not receive significantly higher ratings than either of the DC or EV categories.
These differences reflect an inherent contradiction underlying the concept of half cadence, namely, that a dominant, which functions as the penultimate harmony in an authentic cadential progression, can appear as the ultimate harmony in a cadential progression and thus serve as a satisfactory goal. To be sure, a number of scholars have implicitly supported what we have elsewhere called the 1-Schema model of cadential closure (Sears, in press), in which a number of parameters located within the cadential progression may activate a schematic representation of authentic cadential closure during music listening (Latham, 2009; Meyer, 1973; Schmalfeldt, 1992). The 1-Schema model assumes that listeners generate harmonic and melodic expectations for the moment of cadential arrival, and any deviation at or before cadential arrival results in a violation of listener expectations, and thus would be experienced as a decrease in the cadential strength of the excerpt. In this model the half cadence category therefore represents the weakest cadential category, as a half cadence is marked not by a deviation in the melodic-harmonic content at cadential arrival, but rather by the absence of that content, a theory that may help to explain the ordinal ranking of the cadence categories by the nonmusician group: $\mathrm{PAC}>\mathrm{IAC}>\mathrm{DC}>\mathrm{HC}>\mathrm{EV}$.

During music listening, the 1-Schema model therefore assumes that listeners have no knowledge of the future, and thus, of the material that may occur after the cadential arrival. Yet for a listener aware of the classical style, the material that follows instances of cadential failure differs considerably from the material following the PAC, IAC, or HC categories. By thwarting the expected moment of cadential arrival, theorists typically conceptualize cadential deception and evasion as a kind of derailment. And in order to attain the cadential closure initially promised, the subsequent passage typically features a continuation of an earlier process, sometimes even a direct repetition of the previous cadential progression itself (Schmalfeldt, 1992). In the case of the PAC, IAC or HC categories, however, the subsequent passage typically entails the initiation of a new process (e.g., a new phrase or theme).

Our results may therefore suggest that listeners aware of the classical style have learned to expect the material that typically follows these cadence categories. Differences in the completion ratings for the HC, DC, and EV categories may thus result from a limit in the experimental design. By imposing an artificial boundary at the end of the cadential arrival, our findings do not consider the degree to which the perception of closure may be affected by the material following the cadential arrival. Because listeners continually reevaluate and revise their 
earlier impressions, the surrounding context may be crucial to determining the strength of a given ending.

Moreover, any attempt to model the perception of closure in tonal music must also account for the effect of rhetorical parameters, an approach that traditional definitions of cadence generally do not embrace. The appearance of a dissonance in the melody at cadential arrival, the presence of a cadential trill, the sudden decrease in event density at the cadential arrival, and the temporal duration of the stimulus all significantly affected participants' ratings of completion. Given the exploratory nature of the statistical approach (i.e., a correlational design), however, any future investigations considering the relative importance of these rhetorical features must necessarily adopt a more controlled experimental design. Additionally, by selecting excerpts from a stylistically narrow repertoire-Mozart's piano sonatas-the characteristics that define closure in these excerpts (and thus, the characteristics that may lead to the development of learned schemata), may be idiomatic to this composer and genre. Indeed, currently, relatively little is known regarding the degree to which listeners internalize conventional closing patterns that appear in other style periods or genres (e.g., romantic, rock, jazz).

Finally, in addition to those parameters at local time scales for which the concept of closure seems most apparent (e.g., melody, harmony, rhythm, meter), music analysts also frequently apply the idea to parameters appearing at increasingly global levels of musical organization (e.g., tonality and form). As a result, the question as to whether listeners can perceive tonal closure across global time spans has received a great deal of attention in the scholarly community (Cook, 1987; Gjerdingen, 1999; Marvin, 1999; Spitzer, 2000). However, the degree to which cadences and other ending patterns may close entire formal sections (e.g., a sonata-form exposition)a claim frequently made by music theorists (see Caplin, 2004, pp. 60-61) - was not examined in the present study. In her dissertation examining the effect of global cues on the perception of closure, Joichi (2006) asked participants to rate the degree of completion for musical segments ranging from 4 to 16 measures in length. She reported that the duration of the preceding context significantly affected participant ratings of completion. In the context of our stimuli, the temporal duration of the stimulus also significantly affected the completion ratings, with longer excerpts receiving higher ratings. However, the extent to which this claim may be generalized to longer musical segments (e.g., sonata-form expositions, entire movements) remains unclear. Additionally, a number of other issues concerning the perception of closure provide avenues for further inquiry: for example, the effect of cadential elision (i.e., an event that simultaneously closes the preceding process and initiates the subsequent process), or variations in timing and dynamics surrounding the cadential arrival (i.e., characteristics of performance).

Nevertheless, one essential goal in selecting the stimuli was to explore the effect of cadential failure on the perception of closure, an issue which, to our knowledge, has yet to be considered in the experimental literature. Techniques for cadential deviation are a prevalent feature of the classical style and serve an important formal and expressive function. Instances of cadential failure could therefore provide ideal stimuli for future studies adopting a priming paradigm, as cadential deviations represent a violation of expectation when the listener's expectations are highest (Meyer, 1956). At present a number of priming studies employ cadential progressions in the expected condition, but introduce a Neapolitan chord at cadential arrival in the unexpected condition, a choice which, while syntactically incongruous, rarely occurs in the classical style (Koelsch et al., 2000; 2002; 2003; 2005; Leino et al., 2007; Loui et al., 2005; Loui \& Wessel, 2007; Maess et al., 2001). Yet, instances of cadential deception and evasion provide ready examples of expectancy violation derived from real music.

\section{Author Note}

This research was conducted at the Centre for Interdisciplinary Research in Music, Media, and Technology (CIRMMT). Funding was provided by a Richard H. Tomlinson fellowship and a Quebec doctoral fellowship from the Programme de bourses d'excellence pour étudiants étrangers awarded to David Sears, a Canadian Social Sciences and Humanities Research Council grant and James McGill Professorship awarded to William E. Caplin, and Canadian Natural Sciences and Engineering Research Council and Social Sciences and Humanities Research Council grants and Canada Research Chair awarded to Stephen McAdams. The authors would like to thank Bennett Smith for the design of the interface used in this experiment and the anonymous reviewers for their insightful comments.

Correspondence concerning this article should be addressed to David Sears, Department of Music Research, McGill University, 555 Sherbrooke Street West, Montreal, QC, Canada H3A 1E3. E-mail: david.sears@mail.mcgill.ca 


\section{References}

Aarden, B. (2003). Dynamic melodic expectancy (Unpublished doctoral dissertation). Ohio State University, Columbus, Ohio.

$\rightarrow$ Agawu, K. (1987). Concepts of closure and Chopin's Opus 28. Music Theory Spectrum, 9, 1-17.

Anson-Cartwright, M. (2007). Concepts of closure in tonal music: A critical study. Theory and Practice, 32, 1-18.

$\rightarrow$ Bharucha, J. J. (1987). Music cognition and perceptual facilitation: A connectionist framework. Music Perception, 5, $1-30$.

$\rightarrow$ Bharucha, J. J., \& Krumhansl, C. L. (1983). The representation of harmonic structure in music: Hierarchies of stability as a function of context. Cognition, 13, 63-102.

$\rightarrow$ Bharucha, J. J., \& Stoeckig, K. (1986). Reaction time and musical expectancy: Priming of chords. Journal of Experimental Psychology: Human Perception and Performance, 12, 403-410.

$\rightarrow$ Bigand, E. (2003). More about the musical expertise of musically untrained listeners. Annals of the New York Academy of Sciences, 999, 304-312.

$\rightarrow$ Bigand, E., Madurell, F., Tillman, B., \& Pineau, M. (1999). Effect of global structure and temporal organization on chord processing. Journal of Experimental Psychology: Human Perception and Performance, 25, 184-197.

$\rightarrow$ Bigand, E., \& Parncutt, R. (1999). Perceiving musical tension in long chord sequences. Psychological Research, 62, 237-254.

$\rightarrow$ Bigand, E., \& Pineau, M. (1997). Global context effects on musical expectancy. Perception and Psychophysics, 59, 1098-1107.

Blombach, A. (1987). Phrase and cadence: A study of terminology and definition. Journal of Music Theory Pedagogy, 1, 225-251.

$\rightarrow$ Boltz, M. (1989). Perceiving the end: Effects of tonal relationships on melodic completion. Journal of Experimental Psychology: Human Perception and Performance, 15, 749-761.

Burnham, S. (2002). Form. In T. Christensen (Ed.), The Cambridge history of Western music theory (pp. 880-906). Cambridge, UK: Cambridge University Press.

$\rightarrow$ Caplin, W. E. (1987). The "expanded cadential progression": A category for the analysis of classical form. Journal of Musicological Research, 7, 215-257.

CAPLIN, W. E. (1998). Classical form: A theory of formal functions for the instrumental music of Haydn, Mozart, and Beethoven. New York: Oxford University Press.

Caplin, W. E. (2000). Harmonic variants of the "Expanded Cadential Progression."' In M. Humal (Ed.), A composition as problem II: Proceedings of the Second International Estonian Music Theory Conference (pp. 49-71). Tallinn: Estonian Academy of Music. $\rightarrow$ Caplin, W. E. (2004). The classical cadence: Conceptions and misconceptions. Journal of the American Musicological Society, 57, 51-118.

$\rightarrow$ Cook, N. (1987). The perception of large-scale tonal closure. Music Perception, 5, 197-206.

$\rightarrow$ Corrigall, K. A., \& Trainor, L. J. (2010). Musical enculturation in preschool children: Acquisition of key and harmonic knowledge. Music Perception, 28, 195-200.

$\rightarrow$ Costa-Giomi, E. (2003). Young children's harmonic perception. Annals of the New York Academy of Sciences, 999, 477-484.

$\rightarrow$ Cuddy, L. L., Cohen, A. J., \& Mewhort, D. J. K. (1981). Perception of structure in short melodic sequences. Journal of Experimental Psychology: Human Perception and Performance, 7, 869-883.

$\rightarrow$ Cuddy, L. L., \& Lunney, C. A. (1995). Expectancies generated by melodic intervals: Perceptual judgments of melodic continuinty. Perception and Psychophysics, 57, 451-462.

Eberlein, R. (1997). A method of analysing harmony, based on interval patterns or "Gestalten.” In M. Leman (Ed.), Music, gestalt, and computing (Vol. 1317, pp. 225-236). Berlin: Springer Berlin/Heidelberg.

Eberlein, R., \& Fricke, J. (1992). Kadenzwahrnehmung und Kadenzgeschichte: ein Beitrag zu einer Grammatik der Musik [Cadence perception and the history of the cadence]. Frankfurt am Main: Peter Lang.

Gjerdingen, R. O. (1999). An experimental music theory? In N. Cook \& M. Everist (Eds.), Rethinking music (pp. 161-170). Oxford, UK: Oxford University Press.

Gjerdingen, R. O. (2007). Music in the Galant style. New York: Oxford University Press.

Hatten, R. (1992). Interpreting deception in music. In Theory Only, 12(5), 31-50.

Hepokoski, J., \& Darcy, W. (2006). Elements of sonata theory: Norms, types, and deformations in the late-eighteenth-century sonata. New York: Oxford University Press.

Huron, D. (2006). Sweet anticipation: Music and the psychology of expectation. Cambridge, MA: MIT Press.

ISO 389-8. (2004). Acoustics: Reference zero for the calibration of audiometric equipment-Part 8: Reference equivalent threshold sound pressure levels for pure tones and circumaural earphones [Technical Report]. Geneva, Switzerland: International Organization for Standardization.

$\rightarrow$ Janata, P. (1995). ERP measures assay the difference of expectancy violation of harmonic contexts in music. Journal of Cognitive Neuroscience, 7, 153-164.

JoIchi, J. (2006). Closure, context, and hierarchical grouping in music: A theoretical and empirical investigation (Unpublished doctoral dissertation). Northwestern University, Evanston, Illinois. 
$\rightarrow$ Jonaitis, E., \& Saffran, J. (2009). Learning harmony: The role of serial statistics. Cognitive Science, 33, 951-968.

$\rightarrow$ Justus, T., \& Bharucha, J. (2001). Modularity in musical processing: The automaticity of harmonic priming. Journal of Experimental Psychology: Human Perception and Performance, 27, 1000-1011.

$\rightarrow$ Koelsch, S., Fritz, T., Schulze, K., Alsop, D., \& Schlaug, G. (2005). Adults and children processing music: An fMRI study. NeuroImage, 25, 1068-1076.

$\rightarrow$ Koelsch, S., Grossmann, T., Gunter, T. C., Hahne, A., Schröger, E., \& Friederici, A. D. (2003). Children processing music: Electric brain responses reveal musical competence and gender differences. Journal of Cognitive Neuroscience, 15, 683-693.

$\rightarrow$ Koelsch, S., Gunter, T., Friederici, A. D., \& Schröger, E. (2000). Brain indices of music processing: "Nonmusicians" are musical. Journal of Cognitive Neuroscience, 12, 520-541.

$\rightarrow$ Koelsch, S., Schmidt, S. B.-H., \& Kansok, J. (2002). Effects of musical expertise on the early right anterior negativity: An eventrelated brain potential study. Psychophysiology, 39, 657-663.

Krumhansl, C. L. (1990). Cognitive foundations of musical pitch. New York: Oxford University Press.

$\rightarrow$ Krumhansl, C. L., Bharucha, J. J., \& Kessler, E. J. (1982). Perceived harmonic structure of chords in three related musical keys. Journal of Experimental Psychology: Human Perception and Performance, 8, 24-36.

$\rightarrow$ Krumhansl, C. L., \& Kessler, E. (1982). Tracing the dynamic changes in perceived tonal organization in a spatial representation of musical keys. Psychological Review, 89, 334-368.

Latham, E. (2009). Drei Nebensonnen: Forte's linear-motivic analysis, Korngold's Die Tote Stadt, and Schubert's Winterreise as visions of closure. Gamut, 2, 299-345.

$\rightarrow$ Leino, S., Brattico, E., Tervaniemi, M., \& Vuust, P. (2007). Representation of harmony rules in the human brain: Further evidence from event-related potentials. Brain Research, 1142, 169-177.

$\rightarrow$ Loui, P., Grent-'T-Jong, T., Torpey, D., \& Woldorff, M. (2005). Effects of attention on the neural processing of harmonic syntax in Western music. Cognitive Brain Research, 25, 678-687.

$\rightarrow$ Loui, P., \& Wessel, D. (2007). Harmonic expectation and affect in Western music: Effects of attention and training. Perception and Psychophysics, 69, 1084-1092.

Maess, B., Koelsch, S., Gunter, T. C., \& Friederici, A. D. (2001). Musical syntax is processed in Broca's area: An MEG study. Nature Neuroscience, 4, 540-545.

$\rightarrow$ Margulis, E. H., \& Levine, W. H. (2006). Timbre priming effects and expectation in melody. Journal of New Music Research, 35, 175-182.

Martin, F. N., \& Champlin, C. A. (2000). Reconsidering the limits of normal hearing. Journal of the American Academy of Audiology, 11, 64-66. $\rightarrow$ Marvin, E. W. (1999). The effect of modulation and formal manipulation on perception of tonic closure by expert listeners. Music Perception, 16, 389-408.

Meyer, L. (1956). Emotion and meaning in music. Chicago, IL: University of Chicago Press.

Meyer, L. (1973). Explaining music: Essays and explorations. Berkeley, CA: University of California Press.

Meyer, L. B. (1989). Style and music. Philadelphia, PA: University of Pennsylvania Press.

Miles, J., \& Shevlin, M. (2001). Applying regression and correlation. London: Sage Publications.

NARMOUR, E. (1989). The analysis and cognition of basic melodic structures: The implication-realization model. Chicago, IL: University of Chicago Press.

Narmour, E. (1992). The analysis and cognition of melodic complexity: The implication-realization model. Chicago, IL: University of Chicago Press.

$\rightarrow$ Reber, A. S. (1989). Implicit learning and tacit knowledge. Journal of Experimental Psychology: General, 118, 219-235.

$\rightarrow$ Rohrmeier, M., Rebuschat, P., \& Cross, I. (2011). Incidental and online learning of melodic structure. Consciousness and Cognition, 20, 214-222.

$\rightarrow$ Rosch, E. (1975). Cognitive reference points. Cognitive Psychology, 7, 532-547.

$\rightarrow$ Rosner, B., \& Narmour, E. (1992). Harmonic closure: Music theory and perception. Music Perception, 9, 383-412.

$\rightarrow$ Schellenberg, E. G. (1996). Expectancy in melody: Tests of the implication-realization model. Cognition, 58, 75-125.

$\rightarrow$ Schellenberg, E. G. (1997). Simplifying the implicationrealization model of melodic expectancy. Music Perception, 14, 295-318.

$\rightarrow$ Schellenberg, E. G., Bigand, E., Poulin-Charronnat, B., \& Stevens, C. (2005). Children's implicit knowledge of Western music. Developmental Science, 8, 551-566.

$\rightarrow$ Schmalfeldt, J. (1992). Cadential processes: The evaded cadence and the 'one more time' technique. Journal of Musicological Research, 12, 1-52.

$\rightarrow$ Schmuckler, M. A. (1989). Expectation in music: Investigation of melodic and harmonic processes. Music Perception, 7, 109-150.

$\rightarrow$ Schmuckler, M. A. (1997). Expectancy effects in memory for melodies. Canadian Journal of Experimental Psychology, 51, 292-306.

SEARs, D. (in press). The perception of cadential closure. In M. Neuwirth \& P. Bergé (Eds.), What is a cadence? Theoretical and analytical perspectives on cadences in the classical repertoire. Leuven, Belgium: Leuven University Press.

Sloboda, J. A. (1985). The musical mind: The cognitive psychology of music. Oxford, UK: Oxford University Press.

Sмiтн, B. K. (1995). PsiExp: An environment for psychoacoustic experimentation using the IRCAM Musical Workstation. Paper presented at the meeting of the Society for Music Perception and Cognition, Berkeley, CA. 
$\rightarrow$ Spitzer, M. (2000). The retransition as sign: Listener-oriented approaches to tonal closure in Haydn's sonata-form movements. Journal of the Royal Musical Association, 121, 11-45.

$\rightarrow$ Steinbeis, N., Koelsch, S., \& Sloboda, J. A. (2006). The role of harmonic expectancy violations in musical emotions: Evidence from subjective, physiological, and neural responses. Journal of Cognitive Neuroscience, 18, 1380-1393.

$\rightarrow$ Tan, N., Aiello, R., \& Bever, T. G. (1981). Harmonic structure as a determinant of melodic organization. Memory and Cognition, 9, 533-539.

$\rightarrow$ Tillmann, B., Bharucha, J. J., \& Bigand, E. (2000). Implicit learning of tonality: A self-organizing approach. Psychological Review, 107, 885-913.

$\rightarrow$ Tillmann, B., \& Bigand, E. (2001). Global context effect in normal and scrambled musical sequences. Journal of Experimental Psychology: Human Perception and Performance, 27, 1185-1196.

$\rightarrow$ Tillmann, B., Bigand, E., \& Madurell, F. (1998). Local versus global processing of harmonic cadences in the solution of musical puzzles. Psychological Research, 61, 157-174.

$\rightarrow$ Tillmann, B., Janata, P., \& Bharucha, J. J. (2003). Activation of the inferior frontal cortex in musical priming. Cognitive Brain Research, 16, 145-161. $\rightarrow$ Tillmann, B., \& Poulin-Charronnat, B. (2010). Auditory expectations for newly acquired structures. The Quarterly Journal of Experimental Psychology, 63, 1646-1664.

$\rightarrow$ Trainor, L. J., \& Trehub, S. E. (1994). Key membership and implied harmony in Western tonal music: Developmental perspectives. Perception and Psychophysics, 56, 125-132.

Vallières, M. (2011). Beginnings, middles, and ends: Perception of intrinsic formal functionality in the piano sonatas of W. A. Mozart (Unpublished doctoral dissertation). McGill University, Montreal, Canada.

Vallières, M., Tan, D., Caplin, W. E., \& McAdams, S. (2009). Perception of intrinsic formal functionality: An empirical investigation of Mozart's materials. Journal of Interdisciplinary Music Studies, 3, 17-43.

$\rightarrow$ Vos, P., \& Pasveer, D. (2002). Goodness ratings of melodic openings and closures. Perception and Psychophysics, 64, 631-639.

WEBER, R. (1991). The continuous loudness judgement of temporally variable sounds with an "analog" category procedure. In A. Schick, J. Hellbrúck, \& R. Weber (Eds.), Fifth Oldenburg Symposium on Psychological Acoustics (pp. 267-294). Oldenburg: BIS.

Weiser, M. (1992). Rating cadence stability: The effects of chord structure, tonal context, and musical training (Unpublished doctoral dissertation). McMaster University, Hamilton, Canada. 